\section{Análise da distribuição espacial da gravidez adolescente no Município de Belo Horizonte - MG}

\section{Analysis of the spatial distribution of adolescent pregnancy in the city of Belo Horizonte}

Maria José Nogueira'

Bráulio Figueiredo Alves da Silva"

Samuel Moizés Barcelos'

Virgínia Torres Schall'

' Laboratório de Educação em Saúde do Centro de Pesquisa René Rachou da

Fundação Oswaldo Cruz.

"CRISP - Centro de Estudos de Criminalidade e Segurança Pública.

\section{Resumo}

O georreferenciamento dos eventos de saúde ganha relevância na análise e avaliação de riscos à saúde coletiva, na medida em que incorpora variáveis relacionadas com o meio ambiente e com o perfil socioeconômico da população. Partindo da premissa de que a maternidade precoce não acontece de forma aleatória no interior da sociedade, buscou-se identificar a dependência espacial da gravidez na adolescência com aspectos socioeconômicos e de vulnerabilidade social. Assumindo a existência de uma dependência espacial entre esses fatores, foram utilizadas ferramentas do Exploratory Spatial Data Analysis, isto é, técnicas para descrever e visualizar distribuições espaciais, identificar situações atípicas, descobrir padrões de associação espacial, agrupamento de valores semelhantes (clusters) e sugerir regimes espaciais ou outras formas de heterogeneidade espacial. Destaca-se a correlação, estatisticamente significativa, entre vulnerabilidade social e taxa de nascidos vivos para mulheres com idade entre 12 e 19 anos e entre 20 e 29 anos. Constatou-se a presença de conglomerados com altas proporções de mães adolescentes em associação com as piores condições socioeconômicas e uma menor proporção de mães adolescentes em setores de melhores condições. Tal estudo poderá subsidiar a organização e o planejamento das ações e estratégias na área da saúde sexual e reprodutiva para o público jovem e adolescente, sobretudo em regiões de elevada vulnerabilidade econômica e social.

Palavras-chaves: Gravidez na Adolescência. Estudo Ecológico. Políticas Públicas.

Apoio: Fundação de Amparo à Pesquisa do Estado de Minas Gerais (FAPEMIG), Brasil e Conselho Nacional de Desenvolvimento Científico e Tecnológico (CNPq), Brasil

Correspondência: Maria José Nogueira. Centro de Pesquisa René Rachou e Laboratório de Educação em Saúde da Fundação Oswaldo Cruz. Avenida Augusto de Lima, 1715 - Bairro Barro Preto - Belo Horizonte, MG CEP 30190-002. E-mail: maria.nog@cpqrr.fiocruz.br 


\section{Abstract}

Georeferencing of health data gains relevance as the analysis and assessments of risks to public health incorporate variables related to the environment and to the socioeconomic profile of the population. Early motherhood does not occur at random within the society. This study aims to identify the spatial dependence of adolescent pregnancy within socioeconomic aspects and social vulnerability. Presuming the existence of a spatial dependence between these facets, the present work used the Exploratory Spatial Data Analysis. This tool is a collection of techniques which describes and visualizes spatial distributions; identifies atypical circumstances; discovers patterns of spatial association; makes clusters of similar values; and suggests spatial schemes or other forms of spatial heterogeneity. One of the results highlighted is the statistically significant correlation between social vulnerability and the rate of live births for women between 12-19 and 20-29 years of age. The study identified: (a) the presence of clusters with high proportions of teenage mothers associated with poorer socioeconomic conditions; and (b) a decrease in the proportion of teenage mothers in areas of better conditions, in which the highest number of births occurred in the 20-29 years and above 30 age groups. This study may help the organization and planning of actions and strategies in the area of sexual and reproductive health for young and adolescent audiences.

Keywords: Pregnancy in adolescence. Ecological study. Public Politics.

\section{Introdução}

Nas últimas décadas vêm se ampliando no Brasil a utilização de bancos de dados originados pelos serviços de saúde, como subsídio para a elaboração de políticas de saúde, planejamento e gestão de serviços ${ }^{1}$. Neste contexto, o georreferenciamento dos eventos de saúde torna-se um fator importante na análise e avaliação de riscos à saúde coletiva, e vários trabalhos evidenciam a aplicabilidade das análises espaciais para a compreensão da distribuição espacial dos eventos em saúde e sua relação com os determinantes socioeconômicos e ambientais ${ }^{2-4}$.

A compreensão de como um contexto afeta a saúde de grupos populacionais por meio de seleção, distribuição, interação, adaptação e outras respostas, requer medir seus efeitos em nível de grupo, uma vez que medidas em nível individual não podem dar conta destes processos ${ }^{2,5,6}$. Padrões de mortalidade ou morbidade, propagação de epidemias, transmissão sexual de doenças ou a transferência de comportamentos ou valores não podem ser explicados sem uma abordagem que, além de considerar os grupos, estude o espaço e o tempo. Variáveis como raça,etnia, idade, estado civil, escolaridade e gênero, entre outras, são de suma importância para análise e compreensão dos fenômenos ligados à saúde das populações.

No Brasil, a implantação do Sistema de Informações sobre Nascidos Vivos (SINASC) permitiu a elaboração e implementação de indicadores de saúde materno e infantil além de potencializar as análises que visam estabelecer a relação de tais indicadores com variáveis sociodemográficas. Não obstante, inúmeros estudos procuram traçar o perfil epidemiológico dos nascidos vivos, conhecer o perfil da morbimortalidade da população materno-infantil, bem como analisar fenômenos específicos como gravidez e, principalmente, a gravidez na adolescência ${ }^{7,8}$.

No que diz respeito ao fenômeno da gravidez adolescente, uma série de fatores tem sido associada às suas causas e conseqüências, tornando o seu estudo ainda mais complexo. Entre o universo de variáveis 
relacionadas aos fatores sociais, ambientais, individuais e culturais são predominantes os indicadores de raça/etnia, status socioeconômico, escolaridade e a faixa etária da mãe. Isto se torna mais importante quando se assume a não aleatoriedade espacial da gravidez adolescente no ambiente urbano. A gravidez, assim como outros fenômenos sociais difere-se por regiões e grupos sociais ${ }^{9-11}$.

A parentalidade na adolescência repercutirá de forma distinta segundo a inserção socioeconômica dos indivíduos ${ }^{12,13}$. No estudo de Heilborn et al. (2002) ${ }^{12}$, as autoras destacam que nas classes populares é mais notória a mudança de status dos jovens pais: "simbolicamente, há neste contexto certa ruptura a partir do evento da gravidez". Nas camadas médias, "a parentalidade na adolescência não altera o curso de vida”. Este fato pode até mesmo indicar certo determinismo da gravidez na adolescência nas camadas sociais menos abastadas, uma vez que neste ambiente social a gravidez implica em um mecanismo válido para conduzir à mobilidade social ${ }^{14,15}$.

O estudo de César et al., (2000) ${ }^{16}$ aponta para uma relativização do efeito idade quando se considera o status socioeconômico dos indivíduos. A autora realizou pesquisa em Belo Horizonte para testar a hipótese, segundo a qual a maior mortalidade neonatal de crianças filhas de mães adolescentes está relacionada ao baixo status socioeconômico destas mães, e não à idade das mesmas. Os aspectos fisiológicos que determinariam uma gravidez de alto risco entre adolescentes, tais como peso, altura, estado nutricional e desenvolvimento do aparelho reprodutivo, são agravados pelas condições socioeconômicas e culturais nas quais a adolescente está inserida.

Partindo da premissa de que a maternidade precoce não acontece de forma aleatória no interior da sociedade, mas é determinada por fatores econômicos, sociais e culturais, o objetivo do presente estudo é, a partir dos dados do SINASC, identificar uma dependência espacial da gravidez na adolescência com aspectos socioeconômicos e de vulnerabilidade social.

\section{Metodologia}

\section{Local de investigação}

Este estudo foi realizado no município de Belo Horizonte, Minas Gerais, que, de acordo com o IBGE ${ }^{17}$, em 2001 contava com uma população de 2.238.526 habitantes, distribuídos em uma área de $330,23 \mathrm{~km}^{2}$. A organização territorial dos serviços de saúde do município encontrava-se, em 2006, subdividida em nove distritos sanitários, que correspondem às administrações regionais da Prefeitura de Belo Horizonte e têm definido certo espaço geográfico, populacional e administrativo. São 144 unidades básicas de saúde, cujas áreas de abrangência (129) são estabelecidas com base nos 2.564 setores censitários definidos pelo IBGE.

\section{Fonte de dados}

No Brasil existem poucas fontes de dados sociodemográficos em escala intra-urbana, com exceção do IBGE, que produzem dados nessa escala. No caso das grandes cidades brasileiras, o IBGE coleta as informações demográficas no âmbito dos domicílios e as agrega para disponibilizá-las ao público no nível definido como setor censitário, correspondente a um conjunto de domicílios.

Neste artigo, usamos a base de dados georreferenciada de setores censitários do IBGE para o município de Belo Horizonte relativa ao Censo realizado no ano de 2000. Essa base de dados conta com 2.564 setores censitários que delimitam toda a área da capital mineira e, usando suas informações, foi possível elaborar o Índice deVulnerabilidade Social* IVS - para a cidade e verificar sua distribuição espacial no ambiente em estudo.

\footnotetext{
* Este indicador baseou-se na metodologia utilizada pela Fundação SEADE do Estado de São Paulo para a construção do Índice Paulista de Vulnerabilidade Social, de acordo com a SEADE, que permite uma visão mais detalhada das condições de vida do município. Para maiores detalhes sobre a apresentação dos objetivos principais e utilização prática do Índice de Vulnerabilidade Social ver http://www. al.sp.gov.br/web/ipvs/index_ipvs.htm, acessada por nós em 20 de janeiro de 2007.
} 
O IVS pode ser entendido como o resultado da combinação de duas dimensões, uma socioeconômica e outra demográfica. Os setores censitários e seus residentes são classificados em seis grupos de vulnerabilidade social. Conceitualmente, a dimensão socioeconômica compõe-se da renda apropriada pelas famílias e do poder de geração pelas mesmas, e a dimensão demográfica está ligada ao ciclo de vida familiar. O outro conjunto de dados utilizado neste estudo diz respeito ao número de nascidos vivos no $\mathrm{Mu}$ nicípio de Belo Horizonte no ano de 2005. Os dados do SINASC ${ }^{18}$ foram consolidados pela Gerência de Epidemiologia e Informação da Secretaria Municipal de Saúde de Belo Horizonte (SMS). A partir desta base de dados, foi possível georreferenciar o endereço de residência dos nascidos vivos no ano de 2005, diferenciando-os pela faixa etária da mãe.

\section{Modelo de análise}

Em um primeiro momento, usando os setores censitários como a menor unidade de análise, tratou-se da prevalência da gravidez na cidade de Belo Horizonte. O método mais simples e usual é o cálculo da taxa bruta, que consiste na razão entre o número de eventos ocorridos numa área em um determinado momento do tempo e o número de pessoas-ano expostas à ocorrência deste evento. No entanto, este método está fortemente sujeito à variabilidade entre áreas vizinhas ocasionada pela não existência da ocorrência de eventos em determinado período.

Assim, Castro et. al. ${ }^{19}$ (p.1271), sugerem, como alternativa à utilização da taxa bruta, o uso do Estimador Bayesiano Empírico, "uma das melhores alternativas disponiveis para estimar riscos em pequenas áreas”. Sua aplicabilidade implica no fato de que este estimador faz com que a informação a respeito do risco contida nos dados das outras áreas - por exemplo, de todos os setores censitários de Belo Horizonte - seja usada para estimar o risco em uma dada área, eliminando, assim, a variabilidade contida nas taxas brutas.

Seguindo a proposição contida em Castro et al. ${ }^{19}$, o Estimador Bayesiano Empírico é uma taxa ajustada na forma:

$b_{i}=w_{i} t_{i}+\left(1-w_{i}\right) m$

onde $t_{i}$ é a taxa de risco ou taxa de prevalência de gravidez na área, $m$ é taxa global para a cidade de Belo Horizonte e $w_{i}$ é o peso entre 0 e 1 que depende do tamanho da população da área $i$. Quanto maior a população da área $i$, mais próximo de 1 é o peso $w_{i}$, o que implica que nas áreas cuja população é maior, a estimativa bayesiana empírica é muito próxima da taxa $t_{i}$ e nas áreas com população muito pequena, o valor de $b_{i}$ será intermediário entre $t_{i} \mathrm{e} m^{19}$.

Os dados foram analisados por meios de números absolutos, utilizando-se a população como fator de risco para os eventos de nascidos vivos por faixa etária da mãe. Considerou-se as mulheres na faixa etária de 12 a 19 anos, devido à pouca ocorrência de gravidez entre 10 e 11 anos. As taxas bayesianas empíricas referem-se ao ano de 2005. A unidade de análise utilizada para a correção foram os setores censitários urbanos da cidade de Belo Horizonte e a sua população nesse mesmo ano ${ }^{17}$.

Para facilitar a visualização dos mapas produzidos a partir das taxas bayesianas por setor censitário de Belo Horizonte, procurou-se comparar os mesmos mapas usando a metodologia de mapas de Kernel* ou a interpolação por densidade de Kernel ${ }^{20}$. Sua característica principal é permitir a fácil visualização de grandes volumes de dados pontuais em uma determinada área ou região do mapa.

A estimação da densidade Kernel é uma técnica de interpolação que é apropriada para posições de dados individuais ${ }^{20-22}$. É um método originalmente desenvolvido para obter uma estimativa suavizada de uma densidade de probabilidade univariada ou multivariada a partir de uma

* Usamos o método Normal de Estimativa e o tamanho do bandwidth ou raio foi fixado em 800 metros. 
amostra de dados observados. Estimar a intensidade de um padrão espacial de pontos é muito semelhante a estimar a densidade de probabilidade bivariada, e a estimação de Kernel bivariada pode ser facilmente adaptada para dar uma estimativa da intensidade.

O método pode ser sucintamente descrito da seguinte forma: se $s$ representa uma localização qualquer numa região $R$ e $s_{1}, \ldots, s_{n}$ são as localizações dos $n$ eventos observados, então a intensidade $\lambda(s)$, é estimada por

$\hat{\lambda}_{\tau}(s)=\frac{1}{\delta(s)} \sum_{i=1}^{n} \frac{1}{\tau^{2}} k\left(\frac{\left(s-s_{i}\right)}{\tau}\right)$

Aqui, $k()$ é uma função densidade de probabilidade apropriadamente escolhida, conhecida como kernel, que é simétrica com relação à origem. $O$ parâmetro $\tau>0$ é a largura de banda (bandwidth) e determina a quantidade de suavização - essencialmente é o raio do círculo centrado em $s$ dentro do qual, pontos $s_{i}$ contribuirão significativamente para $\lambda(s)$. O fator abaixo:

$\delta(s)=\int_{R} \frac{1}{\tau^{2}} k\left(\frac{(s-u)}{\tau}\right) d u$

onde $\delta$ é uma correção de fronteiras, justamente o volume sob o kernel centrado em $s$ que fica "dentro" de $R$. O resultado desta estimativa é um valor que representa a densidade de eventos por unidade de áreas. No presente estudo, a estimativa é a densidade do número de nascidos vivos na cidade de Belo Horizonte, no ano de 2005, por quilômetro quadrado.

\section{Procedimentos metodológicos}

O trabalho de consolidação realizado pela Gerência de Epidemiologia e Informação da Secretaria Municipal de Saúde (SMSA) de Belo Horizonte teve como produto uma grande base de dados georrefenciados de nascidos vivos na cidade, em 2005, com exatamente 22.961 registros de nascimentos. Uma análise inicial mostrou que alguns endereços foram citados mais de uma vez no mesmo ano, mostrando que numa mesma localização espacial haviam ocorrido 19 partos. Optou-se por trabalhar apenas com as localizações espaciais em que apareceu apenas uma citação, resultando em um banco de dados com 17.849 ocorrências de nascidos vivos.

Foi realizado o mapeamento dos endereços dos nascidos vivos no município de Belo Horizonte em 2005. Esses endereços foram diferenciados quanto à faixa etária da mãe - até 19 anos, de 20 a 29 anos e mais de 30 anos. A seguir utilizou-se a taxa bayesiana para corrigir o efeito do tamanho da população de risco de maneira a identificar os clusters de nascidos vivos por faixa etária da mãe. Este método minimiza a variabilidade das taxas devida às diferenças entre as áreas do risco subjacente ocasionado pela pura flutuação aleatória ${ }^{23}$.

Para facilitar a visualização dos clusters de nascidos vivos, utilizou-se a interpolação de Kernel como um recurso exploratório para verificar a densidade do evento "nascidos vivos no município de Belo Horizonte no ano de 2005, por faixa etária da mãe". Os mapas de Kernel permitiram identificar as áreas na cidade de Belo Horizonte com maior prevalência de nascidos vivos, por idade da mãe. Esses locais foram identificados em função dos nove distritos sanitários do município.

Buscou-se a correlação estatística entre o status socioeconômico das regiões caracterizado pelo IVS e as taxas bayesianas de nascidos vivos por idade das mães. A unidade de análise considerada são os 2.564 setores censitários urbanos do IBGE, de acordo com o Censo 2000.

$\mathrm{O}$ processamento e a análise dos dados foram realizados com a utilização do software GeoDa 0.9.5.i5 (beta) (Spatial Analysis Laboratory, University of Illinois, UrbanaChanpaign, Estados Unidos), do Statistical Package for the Social Sciences for Windows (SPSS versão 11.0, Chicago, Estados Unidos) e do MapInfo (versão 8.5). O projeto foi aprovado pelos comitês de ética do Centro de Pesquisa René Rachou/FIOCRUZ e da Prefeitura de Belo Horizonte. 


\section{Resultados}

Serão apresentados os resultados referentes às taxas bayesianas de nascidos vivos por faixa etária da mãe e os respectivos mapas de concentração espacial. Posteriormente, apresentam-se os dados da correlação estatística entre o status socioeconômico das regiões caracterizado pelo IVS e as taxas bayesianas de nascidos vivos por idade da mãe.

O Mapa 1 representa a localização espacial dos endereços descritos pelas mães dos nascidos vivos em Belo Horizonte. Existem quase 18 mil pontos neste mapa, inviabilizando qualquer inferência acerca de uma possível concentração em alguma região da cidade. Desse modo, elaborou-se um mapa a partir da estimativa de densidade de Kernel com o objetivo de visualizar e apontar as áreas de maior prevalência de nascidos vivos, representadas no Mapa 2.
Uma análise preliminar aponta uma maior prevalência de nascidos vivos em regiões de vilas e favelas que acumulam uma série de desvantagens socioeconômicas. São aglomerados urbanos de segmentos sociais, vivendo o processo de vulnerabilização social e individual, conseqüência direta de um processo mais amplo que atinge o contexto brasileiro, no qual as tensões ligadas à crise do trabalho geram o desemprego bem como a precarização das condições de trabalho. Tais aspectos, somados à debilidade dos sistemas públicos de proteção social, trazem conseqüências diretas para a qualidade de vida de tais populações.

\section{Taxas corrigidas e estimativa Kernel: mulheres menores de 20 anos}

As duas técnicas utilizadas - taxas corrigidas pelo método bayesiano e estimativa de densidade de Kernel-demonstraram um

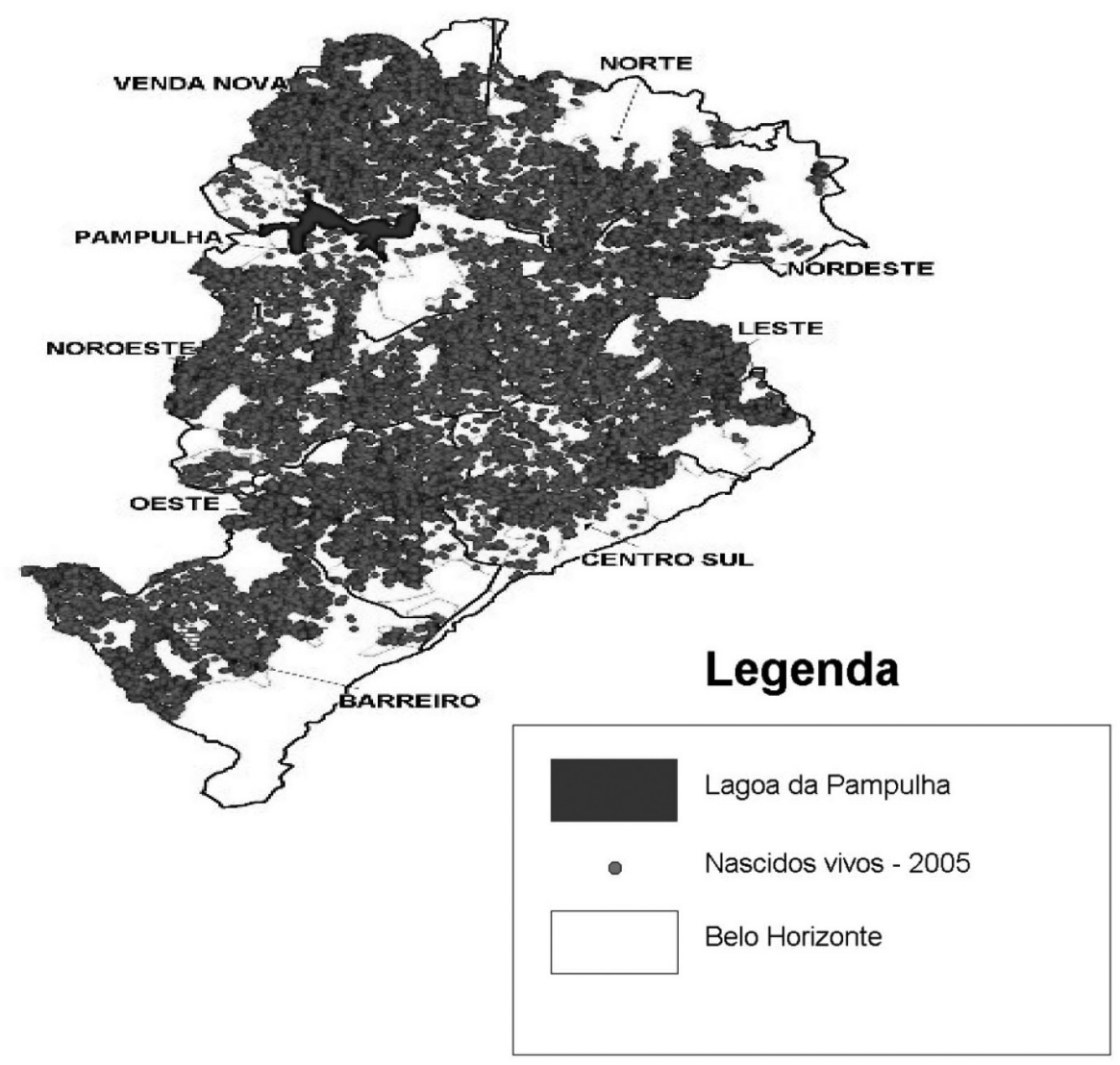

Mapa 1 - Localização espacial dos nascidos vivos em Belo Horizonte, 2005.

Map 1 - Spatial location of live births in Belo Horizonte, 2005. 


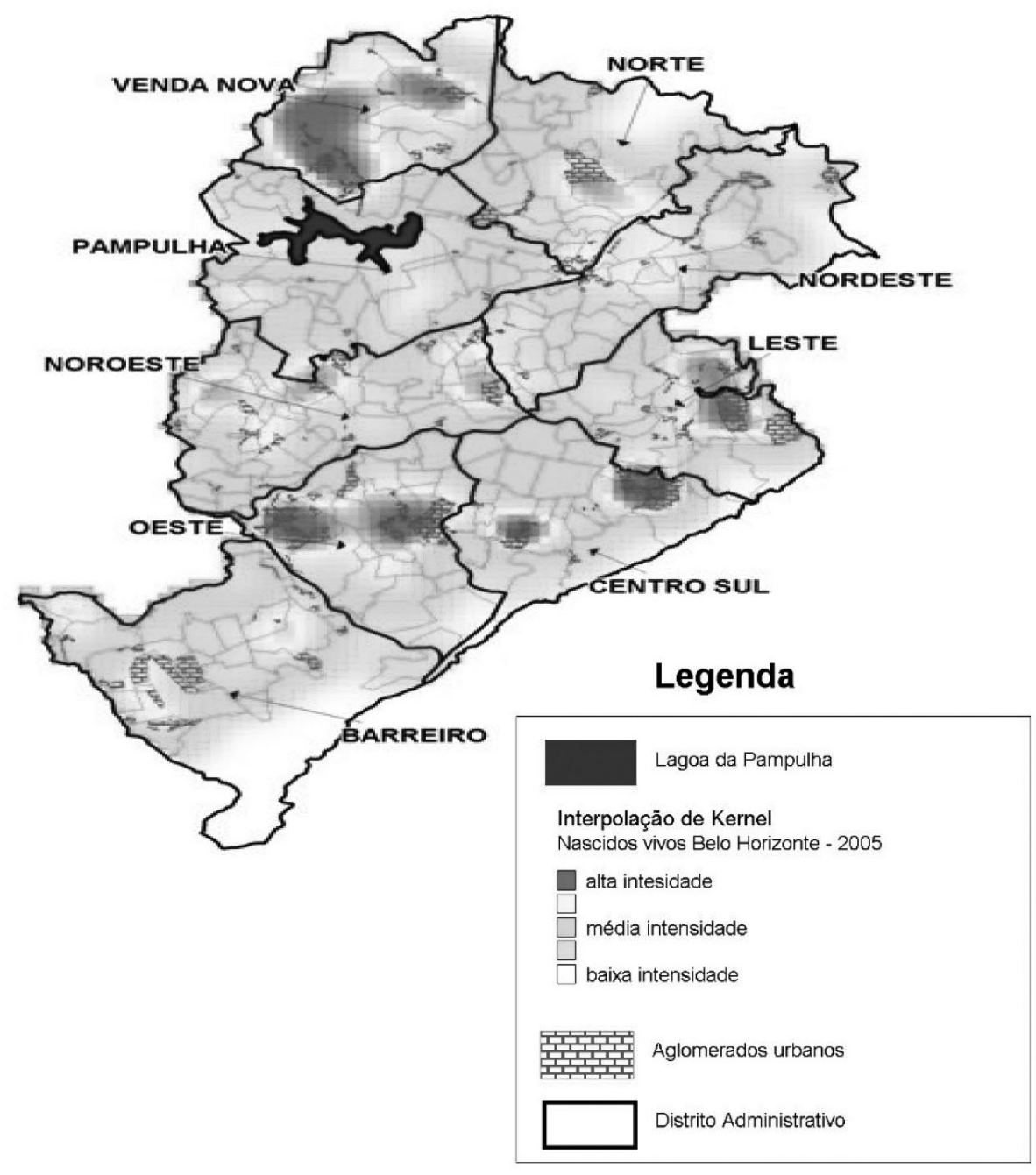

Mapa 2 - Estimação da densidade de Kernel de nascidos vivos em Belo Horizonte, 2005.

Map 2 - Kernel density estimation of live births in Belo Horizonte, 2005.

padrão de concentração espacial distinto na cidade em função da faixa etária da mãe.

O Mapa 3, representativo da prevalência da gravidez em mulheres menores de 20 anos, ou seja, gravidez na adolescência, revela que o fenômeno está concentrado em algumas áreas periféricas do município. Na regional Venda Nova destacam-se o bairro Venda Nova e Mantiqueira. Na Regional Pampulha, que se caracteriza por apresentar grandes contrastes sociais, aparecem dois pontos escuros, na porção centro-sul, referentes à Vila Itamarati e a Vila Ouro Preto. Estas localidades juntamente com o conjunto Lagoa, Chapada do Norte e Engenho Nogueira integram as cinco áreas consideradas de risco nesta regional.

Na regional Nordeste, a grande mancha escura compreende quase a totalidade do bairro Gorduras, que concentra um grande bolsão de miséria, existindo áreas com esgoto a céu aberto, lixo acumulado e aglomerados residenciais do tipo favelas, vilas ou invasão dos sem casa, consideradas de risco elevado e muito elevado. Pode-se notar também outras duas pequenas manchas escuras que representam regiões de grandes áreas favelizadas. Existe uma tendência de diminuição das taxas no interior desta regional à medida que se aproxima de sua porção centro-sul e oeste. O Aglomerado da Serra, constituído por seis vilas, destaca-se 


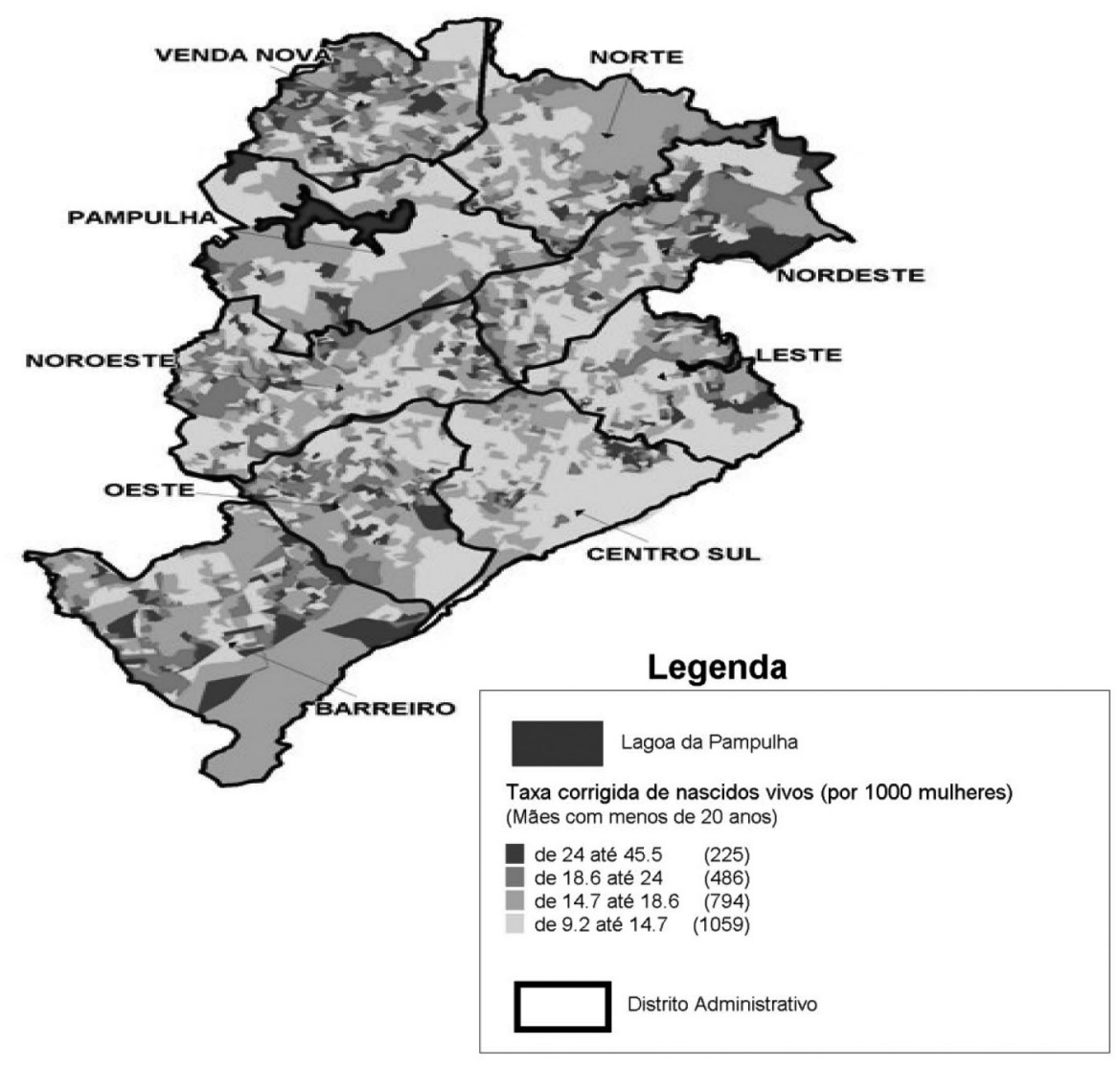

Mapa 3 - Taxa de Nascidos Vivos - Mulheres Menores de 20 anos.

Map 3 - Live birth rate - women under 20 years of age.

na regional Centro Sul por apresentar alta prevalência de gravidez na adolescência. $\mathrm{O}$ aglomerado é o maior conjunto de favelas da região, com a menor renda per capita do município. As vilas diferenciam-se, sobretudo, no tempo de ocupação, no nível de identidade com o espaço físico e social, no nível de organização interna e nas condições socioeconômicas. O Distrito Sanitário Barreiro está localizado na região sudoeste da cidade, e parte de sua população ocupa áreas urbanas de risco, segundo critério socioeconômico sanitário. Nesta regional, as altas taxas aparecem na Vila Cemig, nos bairros Olhos D’Água e Vale do Jatobá.

Complementar às análises bayesianas mostradas no Mapa 3, pode-se observar pela figura do Mapa 4 a concentração espacial de nascidos vivos de mães com idade inferior a 20 anos. Neste mapa é possível identificar áreas com menor concentração espacial de nascidos vivos, representadas pela cor mais suave, e as áreas em que a prevalência de nascidos vivos de mães com idade inferior a 20 anos é maior, áreas de maior concentração espacial - mancha escura no mapa , localizada na região Centro-Sul da cidade.

Como se pode observar, verifica-se uma única área com alta concentração de nascidos vivos de mães com idade inferior a 20 anos na cidade. A mancha escura ocupa quase exclusivamente a região leste da cidade, onde está localizado o Aglomerado da Serra. Comparando-se os dois mapas, verifica-se que nessa região do aglomerado, a Vila Cafezal aparece como uma região de alta prevalência de casos.

Ainda no Mapa 4, na Regional Oeste localizam-se o Aglomerado Cabana do Pai Tomás e o Aglomerado Morro das Pedras que reúnem, atualmente, 25 mil habitantes, os quais possuem um índice de média pre- 


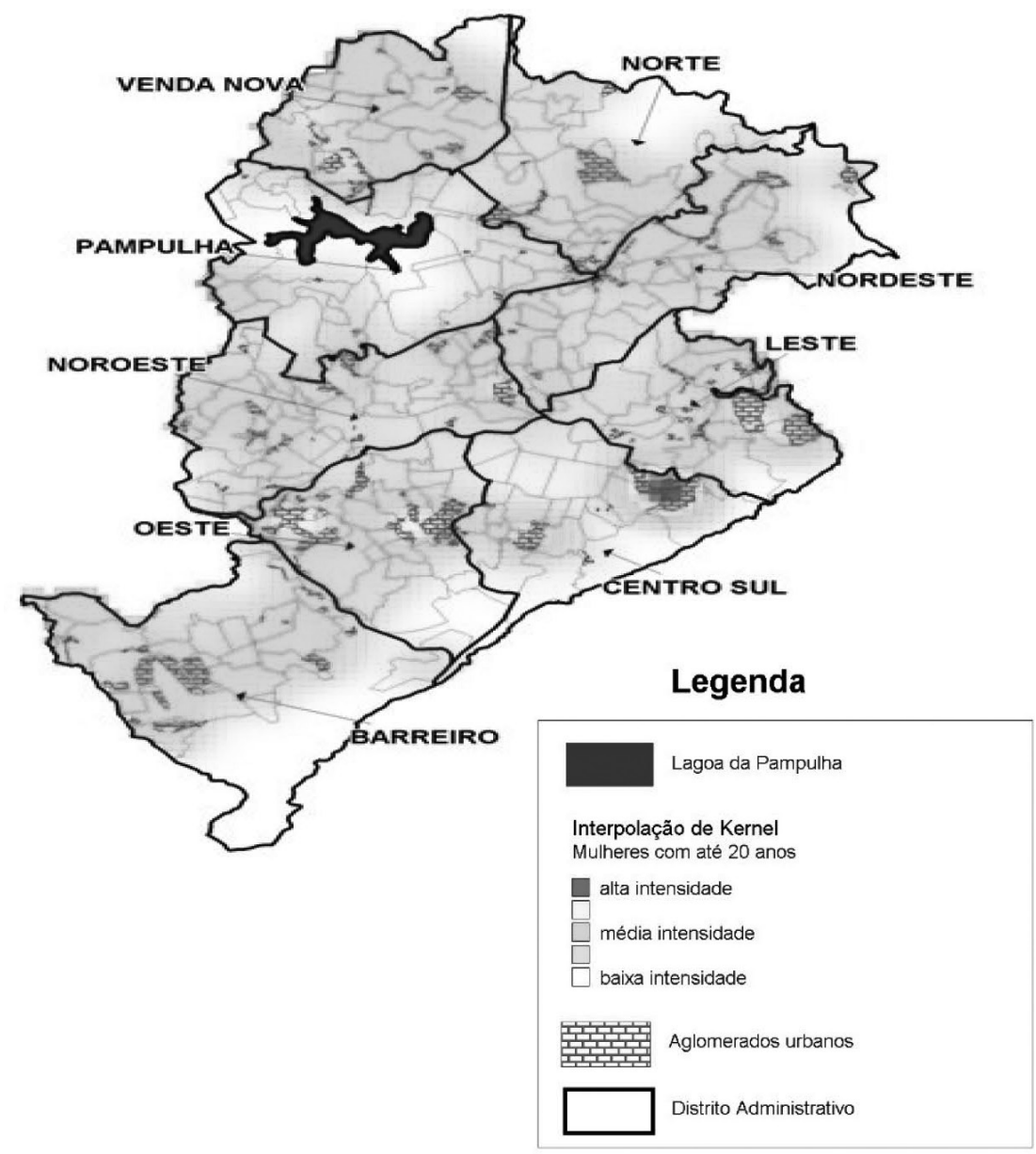

Mapa 4 - Concentração de Nascidos Vivos - Mulheres Menores de 20 anos - BH -2005. Map 4 - Agglomeration of live births - women under age 20 - Belo Horizonte, 2005.

valência de nascidos vivos entre mulheres menores de 20 anos. Todos esses locais são áreas de alta vulnerabilidade social cujos indicadores sociodemográficos apontam para condições precárias de saneamento, infra-estrutura, educação, renda etc.

\section{Taxas corrigidas e estimativa Kernel: mulheres entre e 20 e 29 anos}

As altas taxas de nascidos vivos de mães com idade entre 20 e 29 anos (Mapa 5) concentram-se basicamente na região Norte da cidade: regionais Nordeste, Norte, Venda Nova e Pampulha. Em menor intensidade, outras áreas apresentam taxas elevadas de nascidos vivos, como na região Oeste e Barreiro.
O Mapa 6 indica as concentrações espaciais de nascidos vivos de mães com idade entre 20 e 29 anos. Fica evidente como a elevada prevalência deste fenômeno está concentrada espacialmente nas regiões de aglomerados urbanos (favelas). As regiões escuras e seus arredores que representam, respectivamente, áreas de alta e média prevalência de casos, se sobrepõem às áreas de favelas.

\section{Taxas corrigidas e estimativa Kernel:} mulheres acima de 30 anos

No Mapa 7 nota-se um padrão parecido com o do Mapa 5 e distinto do mapa de gravidez de mães adolescentes (Mapa 3). Na regional Pampulha, chama a atenção o 


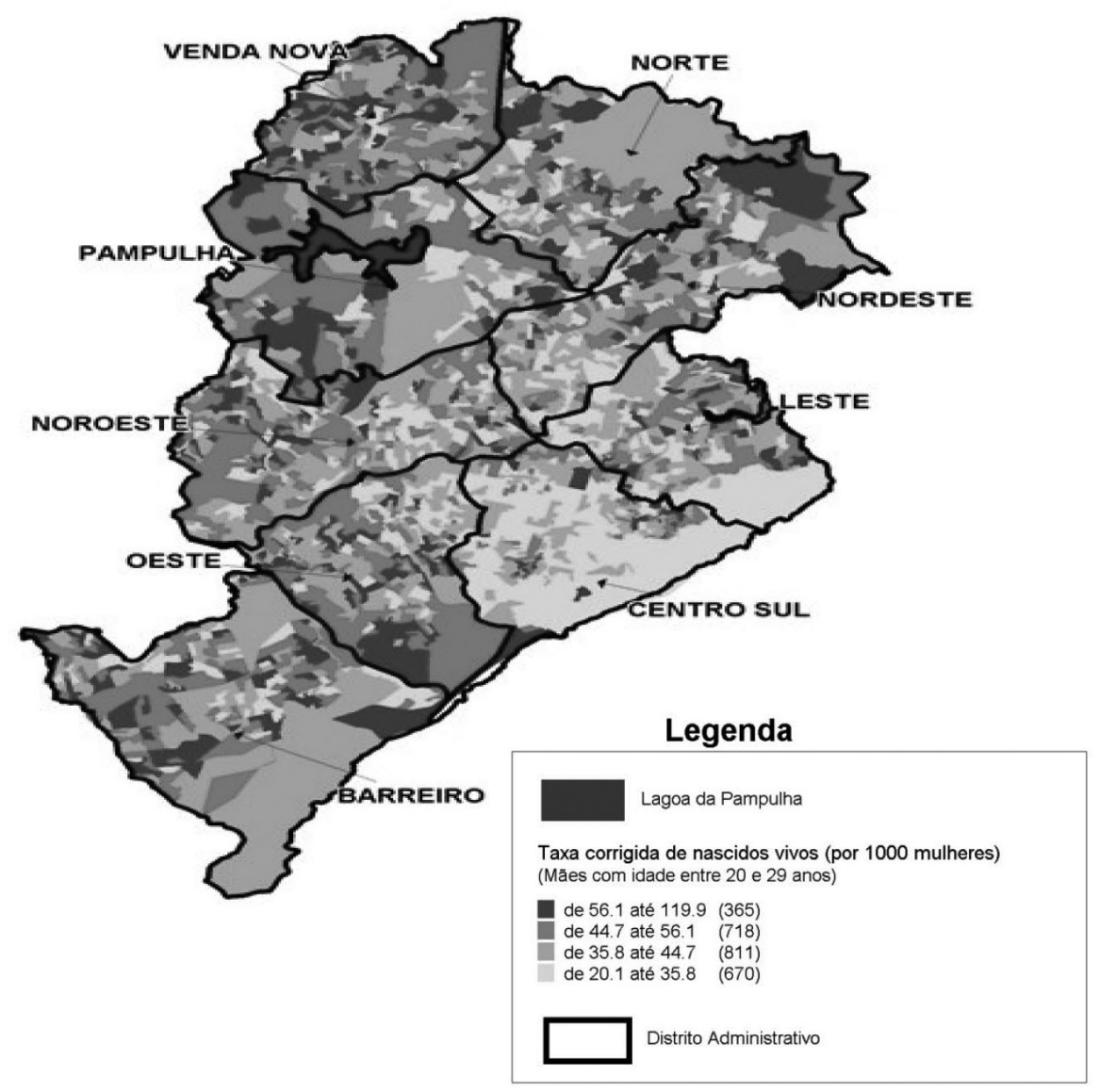

Mapa 5 - Taxa de Nascidos Vivos - Mulheres de 20 a 29 anos.

Map 5 - Live Birth Rate -- women between 20 and 29 years of age.

aparecimento de uma grande área escura. Nesta parte estão localizados os bairros: Xangrilá, Trevo, Céu Azul, Garças e Braúnas, Santa Terezinha, Bandeirantes, Castelo e Paquetá. Os bairros Buritis e Belvedere (áreas escuras) se destacam na Regional Oeste. Na regional Nordeste, a região do Bairro Gorduras evidencia-se em todos os mapas de análise das Taxas de Nascidos Vivos.

A visualização obtida a partir da estimativa de Kernel, representada pelo Mapa 8, aponta uma elevada concentração de nascidos vivos cujas mães têm mais de 30 anos, em quatro localidades: 1) Na Vila do Cafezal, parte do Aglomerado da Serra; 2) entre os bairros Santo Antônio e Sion; 3) no bairro Jardim América e Grajaú; 4) entre os bairros Estrela Dalva e Buritis. Evidencia-se a presença de uma elevada concentração na região do Buritis, bairro caracterizado por uma ocupação recente, apresentando crescimento populacional de mais de $27 \%$ ao ano. Esta região, notadamente, foi ocupada por familias recém formadas, casais jovens pertencentes à classe média, fato que poderia explicar a alta concentração de nascidos vivos de mães com idade superior a 30 anos, uma vez que é recorrente em tais condições a postergação da maternidade bem como a redução de números de filhos por mulher em idade reprodutiva, reflexo do planejamento familiar presente na classe média nas últimas décadas.

\section{Vulnerabilidade Social e Gravidez na Adolescência}

Muitos indicadores podem ser utilizados para medir a pobreza. Fundamentalmente, a questão que se coloca é a necessidade de 


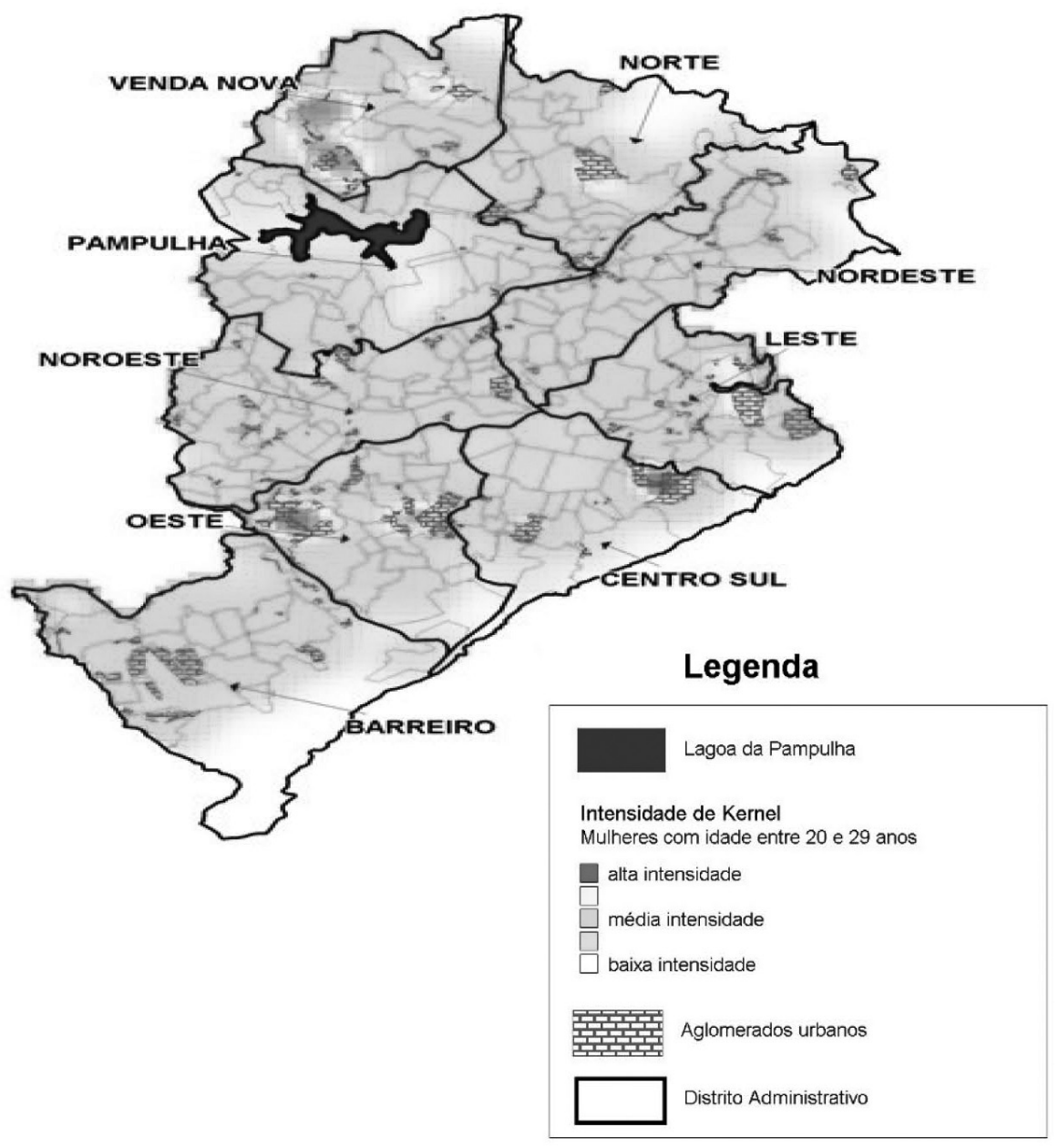

Mapa 6 - Concentração de Nascidos Vivos - Mulheres entre 20 e 29 anos BH - 2005.

Map 6 - Agglomeration of live births - women between 20 and 29 years of age - Belo Horizonte, 2005.

conceituar o fenômeno pobreza para, em seguida, criar medidas e parâmetros de comparação ${ }^{24}$. Neste artigo, utilizou-se o Índice de Vulnerabilidade Social para classificar os 2.564 setores censitários da cidade de Belo Horizonte quanto à dimensão de pobreza, de desvantagem social e cultural, e desigualdade.

Como resultado, conclui-se que pouco mais de $40 \%$ dos setores da cidade possuem nenhuma vulnerabilidade ou vulnerabilidade muito baixa. $31 \%$ dos setores são de vulnerabilidade baixa ou média, e cerca de $25 \%$ dos setores em Belo Horizonte representam áreas de alta ou muito alta vulnerabilidade social. Exatamente 15 setores, o que representa menos de $1 \%$, não possuíam dados e foram desconsiderados na análise.

Notadamente, duas áreas de Belo Horizonte se destacam quanto à concentração de setores considerados com nenhuma vulnerabilidade: a região centro-sul e a região próxima à lagoa da Pampulha. Por outro lado, a vulnerabilidade social em Belo Horizonte se concentra na região periférica da cidade, com alguns pontos no interior que coincidem com os locais de favelas (Mapa 9).

A fim de testar uma possível correlação entre o indicador de vulnerabilidade social e os nascidos vivos por idade da mãe, em Belo Horizonte, essas duas medidas foram tratadas com a mesma distribuição. Como o indicador de vulnerabilidade possui seis ca- 


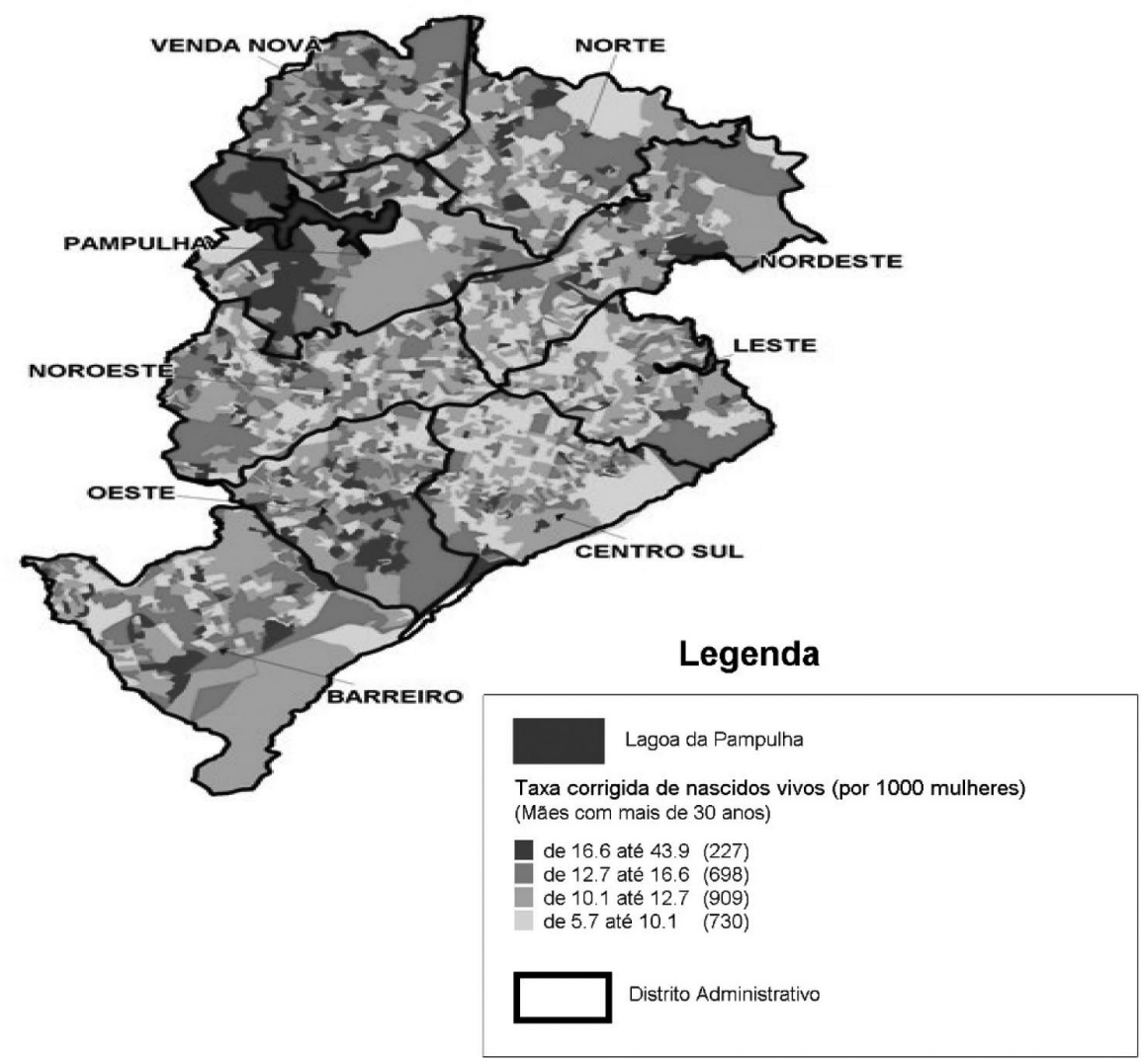

Mapa 7 - Taxa de Nascidos Vivos - Mulheres com mais de 30 anos.

Map 7 - Live birth rate - women above 30 years of age.

tegorias, as taxas de nascimento foram divididas em seis faixas. Em seguida, realizou-se uma análise de correlação de Pearson entre essas variáveis, cujos resultados podem ser visualizados na Tabela 1.

Embora não muito elevada, identifica-se uma correlação, estatisticamente significativa, entre a vulnerabilidade social e a taxa de nascidos vivos para mulheres com idade entre 12 e 19 anos e entre 20 e 29 anos. Essa relação é positiva, indicando que quanto maior é a vulnerabilidade social em um dado setor censitário de Belo Horizonte, maior é a sua taxa de nascidos vivos para as faixas etárias das mães descritas anteriormente. Por outro lado, o IVS não apresentou uma relação estatisticamente significativa para a taxa de nascidos vivos cujas mães possuem mais de 30 anos. Embora esta relação tenha sido negativa, oposto do observado com respeito às outras faixas etárias, não se pode afirmar com segurança estatística que esta relação seja significante. Neste sentido, pode-se inferir que outros fatores alheios à vulnerabilidade social determinam a gravidez de mulheres mais velhas.

\section{Discussão}

Constatou-se a presença de conglomerados com altas taxas de mães adolescentes em associação com piores condições socioeconômicas. Alguns estudos na área apontam resultados semelhantes ${ }^{7,8}$. Quando tal variável é introduzida no estudo do fenômeno da gravidez na adolescência, percebe-se que o fator idade (10 a 19 anos) deixa de ser o principal problema no que diz respeito às conseqüências de uma gravidez precoce; e que o status socioeconômico dos pais da adolescente grávida influenciará aspectos fundamentais: acesso à saúde, pré-natal, condições de educação para as crianças, dentre outros. $^{9}$ 


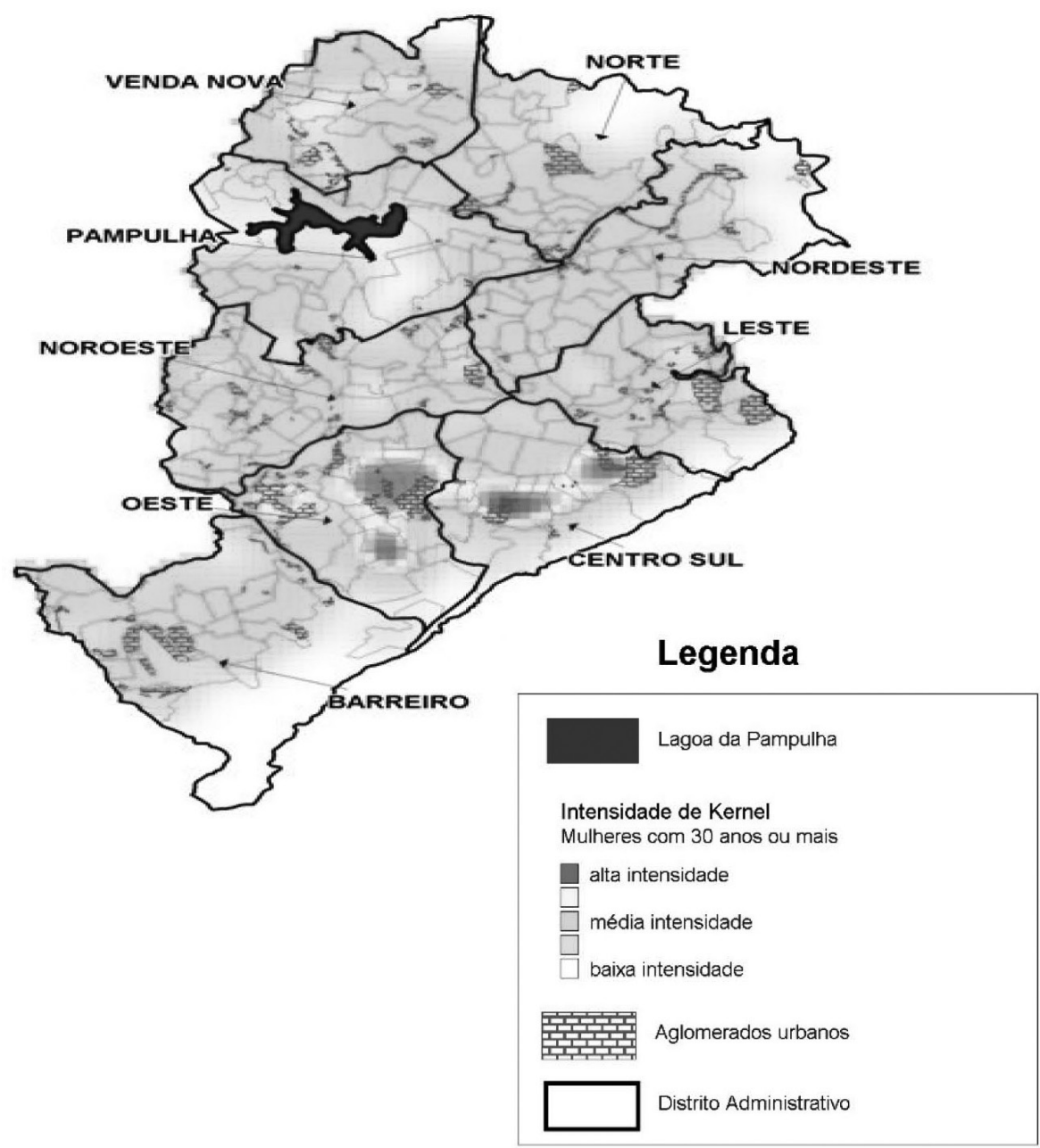

Mapa 8 - Concentração de Nascidos Vivos Mulheres acima de 30 anos - BH - 2005.

Map 8 - Agglomeration of live births - women above age 30 - Belo Horizonte, 2005.

Destaca-se a diminuição da proporção de mães adolescentes em setores de melhores condições, nos quais o maior número de nascimentos ocorre nas faixas de 20 a 29 anos e acima de 30 anos. Tal fato pode estar associado ao projeto de vida e às oportunidades disponíveis às adolescentes de acordo com seu lugar na estrutura social. Um projeto modernizante de identidade feminina assentada na autonomia e na profissionalização estaria mais disponível para as adolescentes das classes médias e altas, ocorrendo a postergação da maternidade bem como a diminuição do número de filhos nesses estratos ${ }^{15}$.

O quadro que se apresenta relativo à distribuição espacial dos nascidos de mães adolescentes é bastante parecido com o padrão encontrado para o número de homicídios de adolescentes. No caso de Belo Horizonte, nas áreas com piores condições socioeconômicas, que apresentam um quadro de vulnerabilidade social e individual, os riscos sociais são cumulativos e fortemente associados às taxas de homicídios e de tráfico de drogas. São regiões que agregam um conjunto significativo de problemas sociais, tais como baixos níveis de escolaridade, domicílios precários, baixa renda, exposição a riscos ambientais, dentre outros ${ }^{25}$. Estudos sobre a chamada violência urbana, por exemplo, têm enfatizado a forte correlação entre as taxas de incidência de homicídios e a 


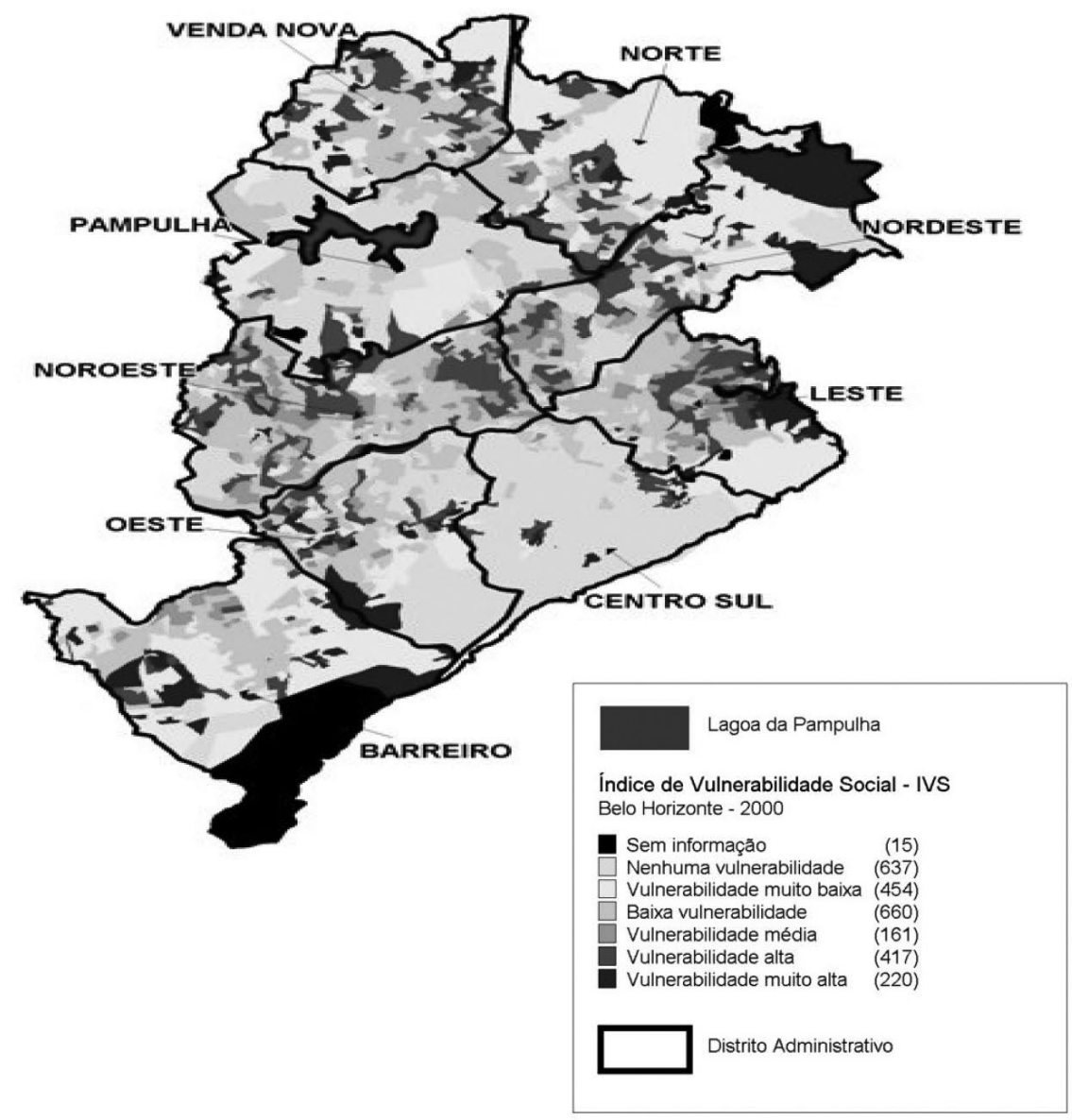

Mapa 9 - Distribuição espacial do Índice de Vulnerabilidade Social no município de Belo Horizonte em 2005.

Map 9 - Spatial distribution of Social Vulnerability Index in the municipality of Belo Horizonte, MG.

Tabela 1 - Correlação de Pearson entre o Indicador de Vulnerabilidade Social e os nascidos vivos por idade da mãe - BH 2005.

Table 1 - Pearson's Correlation between the Social Vulnerability Index and live births by mother's age - Belo Horizonte 2005.

\begin{tabular}{llcccc}
\hline & & ivs & fx_12_19 & fx_20_29 & fx_30mais \\
\hline ivs & Pearson Correlation & 1 & $.265^{* *}$ & .273 & -.002 \\
& Sig. (2-tailed) & & .000 & .000 & .901 \\
& $\mathrm{~N}$ & 2549 & 2549 & 2549 & 2549 \\
\hline fx_12_19 & Pearson Correlation & $.265^{* *}$ & 1 & $.317^{* *}$ & $.131^{* *}$ \\
& Sig. (2-tailed) & .000 & & .000 & .000 \\
& $\mathrm{~N}$ & 2549 & 2564 & 2564 & 2564 \\
\hline fx_20_29 & Pearson Correlation & $.273^{* * *}$ & $.317^{* *}$ & 1 & $.231^{* *}$ \\
& Sig. (2-tailed) & .000 & .000 & & .000 \\
& $\mathrm{~N}$ & 2549 & 2564 & 2564 & 2564 \\
\hline fx_30mais & Pearson Correlation & -.002 & $.131^{* *}$ & $.231^{* *}$ & 1 \\
& Sig. (2-tailed) & .901 & .000 & .000 & \\
& $\mathrm{~N}$ & 2549 & 2564 & 2564 & 2564 \\
\hline
\end{tabular}


precariedade urbana. Na maioria dos setores censitários que compõem tais áreas, o índice de vulnerabilidade à saúde é alto, apontando para a necessidade de refletir sobre a interface entre condições de vida, pobreza e saúde. Tal temática já é recorrente nos estudos contemporâneos que tentam incorporar novos referenciais analíticos e abordagens interdisciplinares para a reflexão conceitual e metodológica sobre pobreza, desigualdades sociais e saúde ${ }^{26}$.

\section{Considerações finais}

Embora a paridade na adolescência esteja relacionada ao status socioeconômico, representado pelo IVS, destaca-se que o fenômeno não é unicausal e suas repercussões no âmbito individual e social são distintas. A aceitação e o apoio das famílias, o planejamento da criança, a coabitação ou não com o parceiro e a presença de instrumentos públicos de saúde e educação para as mães e seus filhos são fundamentais para configurar o cenário de ocorrência da gravidez. $\mathrm{O}$ entendimento das questões culturais e de fatores socioambientais que ocorrem no quadro da vulnerabilidade indica que as políticas que queiram atuar para alterar esse quadro não devem se pautar na perspectiva homogeneizante das categorias adolescentes e gravidez na adolescência, mas antes compreendê-las como categorias socioconstruídas. Um fator limitante neste estudo refere-se ao nível de agregação da faixa etária das mães adolescentes (12 a 19 anos). Acredita-se que o estudo dos dados desagregados em faixas de 12 a 14 e 15 a 19 anos possibilita um melhor entendimento do fenômeno, uma vez que as realidades dos adolescentes nessas faixas etárias são distintas. As causas, as conseqüências e o posicionamento frente à situação da maternidade e paternidade se apresentarão com singularidades em cada contexto.

Em outro aspecto, o enfrentamento do fenômeno requer políticas que ultrapassem o recorte setorial e busquem ações integradas com maior potencial para alterar o quadro de vulnerabilidades. Tal estudo poderá subsidiar a organização e o planejamento das ações e estratégias na área da saúde sexual e reprodutiva para o público jovem e adolescente no município de Belo Horizonte.

\section{Referências}

1. Bittencourt AS, Camacho LAB, Leal MC. O Sistema de Informação Hospitalar e sua aplicação na saúde coletiva. Cad Saúde Pública 2006; 22(1): 19-30.

2. Bailey T. Spatial statistics methods in health. Cad Saúde Pública 2001; 17: 1083-98.

3. Friche AAL, Caiaffa WT, César CC, Goulart LMF. Qualidade dos dados do Sistema de Informação sobre Nascidos Vivos - SINASC - em Belo Horizonte, 1999. In: VI Congresso Brasileiro de Epidemiologia, 2004. Recife: ABRASCO.

4. Opromolla PA, Dalben I, Cardin M. Análise da distribuição espacial da hanseníase no Estado de São Paulo, 1991-2002. Rev Bras Epidemiol 2005; 8(4): 356-64.

5. Susser, M. The logic in the ecological II. The logic of design. Am J Public Health 1994; 84: 830-5.

6. Diez-Roux AV. Bringing context back into epidemiology: variables and fallacies in multilevel analysis. Am J Public Health 1998; 88(2): 216-22.
7. D’Orsi E, Carvalho MS. Perfil de nascimentos no Município do Rio de Janeiro: uma análise espacial. Cad Saúde Pública 1998; 14: 367-79.

8. Friche AAL, Caiaffa WT, César CC, Goulart LMF, Almeida MCM. Maternal and child health indicators in Belo Horizonte, Minas Gerais State, Brazil, 2001: an analysis of intra-urban differences. Cad Saúde Pública 2006; 22(9): 1955-65.

9. Gama SGN, Scwarcwald CL, Leal MC. Experiência de gravidez na adolescência, fatores associados e resultados perinatais entre puérperas de baixa renda. Cad Saúde Pública 2002; 18: 153-61.

10. Sabroza AR, Leal MC, Gama SGN, Costa JV. Perfil sóciodemográfico e psicossocial de puérperas adolescentes do Município do Rio de Janeiro, Brasil - 1999-2001. Cad Saúde Pública 2004; 20(S1): S112-S20.

11. Simões VM, Silva AA, Bettiol H, Lamy-Filho F, Tonial SR, Mochel EG. Características da gravidez na adolescência em São Luís, Maranhão. Rev Saúde Pública 2003; 37(5): 559-65. 
12. Heilborn ML, Salem T, Rohden, F, Brandão E, Knauth D, Víctora C et al. Aproximações socioantropológicas sobre a gravidez na adolescência. Horiz Antropol 2002; 8(17): 13-45.

13. Esteves JR; Menandro PRM Trajetórias de Vida: repercussões da maternidade adolescente na biografia de mulheres que viveram tal experiência. Estudos de psicologia 2005; 10(3): 363-70.

14. Monteiro S. Gênero, Saúde e Proteção entre Jovens Um perfil tradicional. In: Barbosa RM et AL. (orgs.). Interfaces: Gênero, Sexualidade e Saúde Reprodutiva. Campinas: Editora da Unicamp; 2002. p.23-48.

15. Desser, N. Adolescência: sexualidade e culpa. Rio de Janeiro: Rosa dos Tempos; 1993.

16. César CC, Miranda RP, Abreu DM. Efeito idade ou efeito pobreza? Mães adolescentes e mortalidade neonatal em Belo Horizonte. Rev Bras Estud Popul 2000; 17(1-2): 17796.

17. Instituto Brasileiro de Geografia e Estatística. Censo demográfico. Brasília: IBGE; 2001. Disponível em http:// www.ibge.gov.br/ [Acessado em 10 de dezembro de 2004].

18. Sistema de Informações de Nascidos Vivos. Informações de saúde: nascidos vivos. Brasília: SINASC, 2005. Disponível em http:/ / tabnet.datasus.gov.br/cgi/ deftohtm.exe?sinasc/cnv/ nvmg.def [Acessado em 19 de janeiro de 2007].
19. Castro MSM, Vieira VA, Assunção RM. Space-time analysis of lung cancer mortality in Southern Brazil. Rev Bras Epidemiol 2004; 7(2): 131-43.

20. Bailey TC, Gatrell AC. Interactive Spatial Data Analysis. Essex: Longman; 1995

21. Härdle W. Smoothing Techniques: with Implementation in S. New York: Springer; 1991.

22. Bowman AW, Azzalini A. Applied Smoothing Techniques for Data Analysis: The Kernel Approach with S-Plus Illustrations. Oxford: Oxford University Press; 1997.

23. Assunção RM, Barreto, Sandhi Maria; Guerra, Henrique L; Sakurai, Emília. Mapas de taxas epidemiológicas: uma abordagem Bayesiana. Cad Saúde Pública 1998; 14(4): 713-23.

24. Rocha S. Pobreza no Brasil: afinal, de que se trata? Rio de Janeiro: Editora FGV; 2003.

25. Beato Filho CC, Assunção RM, Silva BFA, Marinho FC, Reis IA, Almeida MCM. Homicide clusters and drug traffic in Belo Horizonte, Minas Gerais State, Brazil from 1995 to 1999. Cad Saúde Pública 2001; 17(5): 1163-71.

26. Magalhães R, Burlandy L, Senna MCM. Pobreza, desigualdades sociais e saúde: desafios para as políticas públicas. Ciênc Saúde Coletiva 2007; 12(6): 1412.

Recebido em: 13/03/09

Versão final reapresentada em: 03/06/09

Aprovado em: 23/06/09 